\title{
Cellular organization and molecular differentiation model of breast cancer-associated fibroblasts
}

\author{
Susann Busch ${ }^{1 *} \mathbb{D}$, Daniel Andersson ${ }^{1}$, Eva Bom², Claire Walsh ${ }^{1}$, Anders Ståhlberg ${ }^{1}$ and Göran Landberg ${ }^{1}$
}

\begin{abstract}
Background: The role of cancer-associated fibroblasts (CAFs) during tumour progression is obscured by the inherently complex, heterotypic nature of fibroblast cells and behaviours in various subtypes of malignancies. Therefore, we sought to identify distinct fibroblast subpopulations at the single-cell level.

Methods: Using single-cell quantitative PCR as a powerful tool to study heterogeneity and rare cell events, in a high-throughput manner a panel of gene targets are run simultaneously on transcripts isolated from single cells obtained by fluorescence-activated cell sort. Assessment of cells with stem-like characteristics was attained by anchorage-independent, anoikis-resistant culture.

Results: Single-cell analysis of fibroblasts and their tumour-activated counterparts demonstrated molecularly distinct cell types defined by differential expression of characteristic mesenchymal and fibroblast activation markers. Identified subpopulations presented overlapping gene expression patterns indicating transitional molecular states during fibroblast differentiation. Using single-cell resolution data we generated a molecular differentiation model which enabled the classification of patient-derived fibroblasts, validating our modelling approach. Remarkably, a subset of fibroblasts displayed expression of pluripotency markers, which was enriched for in non-adherent conditions. Yet the ability to form single-cell derived spheres was generally reduced in CAFs and upon fibroblast activation through TGF $\beta 1$ ligand and cancer cell-secreted factors. Hence, our data imply the existence of putative stem/progenitor cells as a physiological feature of undifferentiated fibroblasts.

Conclusions: Within this comprehensive study we have identified distinct and intersecting molecular profiles defining fibroblast activation states and propose that underlying cellular heterogeneity, fibroblasts are hierarchically organized. Understanding the molecular make-up of cellular organization and differentiation routes will facilitate the discovery of more specific markers for stromal subtypes and targets for anti-stromal therapies.
\end{abstract}

Keywords: Cancer-associated fibroblast, Breast cancer, Tumour stroma, Single-cell analysis

\section{Background}

With the need of more personalized medicine, targeting the tumour microenvironment/stroma has become an increasingly relevant and emerging concept. A permissive or tumour-promoting stroma coevolves with cancer cells during tumourigenesis [1] and in breast cancer, cancer-associated fibroblasts (CAFs) are the most prominent stromal cell type. CAFs thus present an attractive

\footnotetext{
* Correspondence: Susann.Busch@gu.se

'Department of Pathology, Sahlgrenska Cancer Center, Institute of Biomedicine, Sahlgrenska Academy, University of Gothenburg, Gothenburg, Sweden

Full list of author information is available at the end of the article
}

treatment option. Yet, the heterogenic nature and functional complexity of CAFs, although well-known, has been greatly understudied.

Physiologically, fibroblasts are mesenchymal cells and as the main cellular component of connective tissues maintain tissue homeostasis. Fibroblasts deposit and remodel extracellular matrix (ECM) and facilitate wound healing upon injury-induced activation. Transforming growth factor-beta (TGF $\beta$ ) is the most potent inducer of fibroblast transformation into 'activated' fibroblasts with elevated smooth muscle actin-alpha (SMA $\alpha)$ levels (encoded by ACTA2). This myodifferentiation gives rise 
to a contractile and secretory cell, a phenotype which has been associated with CAFs [2, 3]. However, although genetically stable, CAFs display a vast cellular heterogeneity and thus far no unique marker or common predominant pathway has been identified. Classically CAFs are assigned with pro-tumorigenic qualities. Recent advances however have revealed tumour-inhibitory features [4] along with cancer subtype-specific characteristics [5]. This suggests that tumour-residing fibroblasts manifest not only phenotypic but also functional plasticity and therefore necessitating a more extensive understanding of the origin and biology of CAFs.

This study aims to dissect cellular composition constituting fibroblast heterogeneity on single-cell level enabling identification and characterization of molecular subsets of breast cancer-associated fibroblast subpopulations with reference to normal tissue-resident fibroblasts. Single-cell resolution data provided us with indepth information for adequate modelling of fibroblast subsets. As a result, generation of a molecular fibroblast differentiation model enabled us to categorize patientderived fibroblasts regarding its activation state. We therefore report herein proof-of-principle how to identify and characterize individual tumour stroma subtypes. We further provide first evidence of fibroblast stem or progenitor cells signifying a cellular hierarchy.

We have previously hypothesized that the existence of functional and phenotypic diverse fibroblast subpopulations may reflect either [a] different stages during fibroblast activation which may either be transitory or represent irreversible cell types, [b] different cells of origin (such as tissue-resident fibroblasts, recruitment of circulating mesenchymal stem cells or fibrocytes), [c] distinct modes of activation (eg. cancer subtype, chemokine profile or physical tension), [d] a stochastic/ hierarchical organization, or likely [e] a combination of thereof [6]. In line with this and the presented study, we propose that the observed heterogeneity of CAFs is a consequence of transitional molecular states with overlapping marker expression during fibroblast activation with an underlying hierarchical program.

\section{Methods}

\section{Cell culture and fibroblast isolation}

Control fibroblasts and expCAFs cells are a kind gift of Dr Akira Orimo. The generation of these cell lines have been described previously [7]. Fibroblast cell lines were cultured in DMEM with $10 \%$ foetal bovine serum (FBS) in a humid chamber with $5 \% \mathrm{CO}_{2}$ at $37{ }^{\circ} \mathrm{C}$. Primary CAFs were isolated from surgically resected invasive breast carcinomas on the day of surgery. Normalmatched control tissue was taken approximately $2 \mathrm{~cm}$ distant from tumour area. Tumour and normal tissue were mechanically dissected and subjected to explant outgrowth and cultured in DMEM supplemented with $20 \%$ FBS and $1 \%$ non-essential amino acids in a humid chamber at $37{ }^{\circ} \mathrm{C}$ with $5 \% \mathrm{CO}_{2}$ and at a physiological level of $5 \% \mathrm{O}_{2}$. Outgrown cells were trypsinized and filtered through a 100um cell strainer to obtain single-cell suspension for further propagation. Throughout cell culture patient-derived fibroblast cells sequentially outnumbered tumour cells which are sensitive to sequential passaging and epithelial cell clusters disappeared after passage three. Enriched fibroblast cells were cultured up to ten passages. Fibroblastic nature was assessed by microscopic assessment of characteristic spindle-like cell morphology (Additional file 1A). For clinical information of all utilized tumours refer to Additional file 1B.

\section{Single-cell gene expression profiling}

Procedure has been described previously [8]. Briefly, single cells were obtained by fluorescence-activated cell sorting (FACS Aria II, BD Biosciences) into a 96-well plate excluding non-viable (7AAD+) cells and subjected to direct cell lysis in $5 \mathrm{ul}$ containing $1 \mathrm{ug} / \mathrm{ul}$ BSA in DNase/RNase-free water, followed by immediate freezing on dry ice. RNA was reversed transcribed and sort efficiency was monitored by measuring GAPDH. Obtained cDNA was preamplified using a pool of gene-specific primers. Samples were prepared for high-throughput real-time quantitative PCR using Fluidigm platform to assess gene expression levels of selected genes. Obtained single-cell qPCR data was pre-processed and subjected to multivariate analysis using GenEx (Multid Analyses, Version 5) as has been described [9]. No normalization to reference gene was performed, instead gene expression data are presented per cell. For principle component analysis (PCA) data were autoscaled per gene and for unsupervised clustering (heatmaps) data were meancentred by gene using log2-transformed data unless otherwise specified. To compensate for variations in absolute RNA expression levels, data in Fig. 2b were autoscaled by cell which corresponds to a global normalization and standardizes expression values to a common scale. Distribution of ACTA2 gene expression levels were computed in Graphpad Prism (Version 5.01).

\section{Anoikis resistance and sphere formation assay}

Adherent fibroblast cells were trypsinized, washed with PBS and syringed with 25-gauge needle to obtain a single-cell suspension. Triplicates of 5000 cells were seeded in $2 \mathrm{ml}$ phenolred-free DMEM/F12 supplemented with $2 \%$ B27 serum (Gibco) into non-adherent, poly(2-HEMA)-coated 6-well plates. Number of spheres larger than 50um was assessed after 5 to 6 days. Cells were treated with either $10 \mathrm{ng} / \mathrm{ml}$ recombinant human TGF $\beta 1$ (rhTGF $\beta 1$ ), 10uM SB431542 (TGFBR1 inhibitor), 10uM LY2109761 (TGBFR1/TGFBR2 inhibitor), 
LY2157299 (TGFBR1 inhibitor) or $72 \mathrm{~h}$ cell-conditioned media of ER $\alpha$-positive breast cancer cell line MCF7 or $E R \alpha$-negative breast cancer cell line MDA-MB-231 for $48 \mathrm{~h}$ prior to sphere formation assay. Data are shown in mean+/-SEM and two-way analysis of variance (ANOVA) with replicates was performed for statistical analysis using Graphpad Prism (Version 5.01). For anoikis-resistance, single-cell suspension setup was scaled up $20 \mathrm{x}$ to obtain sufficient number of viable cells after $24 \mathrm{~h}$ culture for subsequent RNA analysis.

\section{Label-retention assay}

For PKH26 staining of sphere-forming cells, adherent fibroblast cells were trypsinized, washed with serum-free media, suspended in Diluent $\mathrm{C}$ and labelled with $1 \mathrm{uM}$ PKH26 dye for 3 min according to manufacturer's instruction (Sigma-Aldrich). Stained cells were washed three times, syringed to obtain single cells and counted to seed 500 cells for sphere formation under nonadherent conditions as described in section above.

\section{Standard quantitative RT-PCR}

Following $24 \mathrm{~h}$ anoikis resistance cells were collected, directly lysed and subjected to RNA isolation (Qiagen). RNA was transcribed using Grandscript Reverse Transcriptase (TATAA) and $20 \mathrm{ng}$ of resulting cDNA was used for real-time quantitative PCR (Applied Biosystems 7900) using Sybrgreen (Bioline) and 0.4uM of the same target-specific primers used for single-cell gene expression profiling.

\section{Results}

To delineate cellular heterogeneity of fibroblasts we deployed a microfluidics platform (Fluidigm) for multiplex gene expression analysis of individual cells. This approach allows analysis of simultaneous gene expression and resolves cellular diversity at the single-cell level. We designed single-cell assays to study gene expression typically associated with mesenchymal cells and fibroblast activation alongside genes involved in pluripotency, breast cancer-specific stemness (BCSC), epithelial-tomesenchymal transition, cell cycle and proliferation (see list of target genes in Fig. 1a). Single-cell assays and subsequent quantitative PCR procedure have previously been described by our group [8]. Briefly, we collected single cells using fluorescence-activated cell sorting excluding non-viable cells. Sort efficiency was monitored by measuring cells positive for GAPDH expression to verify $>80 \%$ positivity. Samples were pre-amplified and subjected to high-throughput single-cell qPCR analysis using the Fluidigm platform. Workflow for processing of obtained single-cell data has been reported [9] and was applied accordingly. Only GAPDH-positive samples were included for downstream data analysis. For our study we employed an experimental CAF cell line model (expCAFs) [7] and patient-derived material for descriptive and functional analysis of normal fibroblasts and their breast cancer-activated counterparts (see schematic depiction of study design in Fig. 1b).

\section{Molecular markers characterizing fibroblast activation}

Comparing frequencies of cells expressing relevant genespecific targets demonstrates that fibroblast cells were positive for typical fibroblast markers (such as VIM, $C D 44)$ and mostly negative for epithelial markers (CDH1, EPCAM) (Fig. 1c). Characteristic fibroblast activation gene targets such as $A C T A 2, C O L 1 A 1$ and TNC were significantly upregulated in CAFs together with typical cancer-induced chemokines most prominently CXCL12, which has been reported for this cell line [7]. Concomitantly CAV1 was downregulated in CAFs alongside other mesenchymal markers such as CD44 and VIM. Loss of CAF-specific Caveolin-1 expression has been reported to be linked to worse prognosis in multiple cancers including breast cancer [10].

Based on the individual single-cell gene expression profiles, principle component analysis (PCA) revealed clearly distinguishable clusters of experimental CAFs when compared to their control counterparts (Fig. 1d). Strikingly, corresponding gene clusters were predominantly defined by gene groups (ie. pluripotency, BCSC-like, proliferation, fibroblast and epithelial markers) indicating potential co-regulation of a specific cellular function. However, expression patterns of transcription factors and soluble factors were more diverged, suggesting a more complex transcriptional regulation and induction of paracrine molecules.

SMA $\alpha$ is the most widely used marker to assess CAF activation status and is associated with worse clinical outcome [11]. Interestingly, we observed a bimodal distribution of expression level among the experimental CAF population (Fig. 1e), indicating a mixed population of ACTA2 low and high expressing cells. Thus, in order to determine the role of SMA $\alpha$ in regard to fibroblast activation status we stratified cells according to their ACTA2 expression level for correlation with other CAF activation markers. However, comparison of ACTA2 low to ACTA2 high expressing CAFs demonstrated no difference in gene expression profile other than ACTA2. Yet compared to control fibroblast, $A C T A 2$ low CAFs showed significant higher expression of CXCL12, PDGFA, VEGFA and $A L D H 1 A 3$ (Fig. 1f). These data suggest that CAFs with low SMA $\alpha$ expression do not correspond to a normal fibroblast phenotype but rather constitute a subset of activated CAFs with tumour-promotive features. Whether SMA $\alpha$ induction is in fact abrogated or merely bypassed using alternative fibroblast activation mechanisms needs to be clarified. 

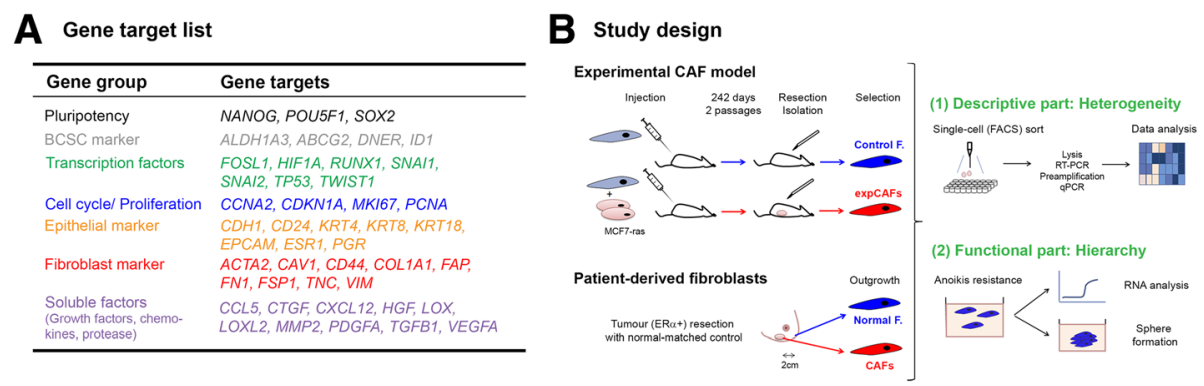

C Frequency and expression level
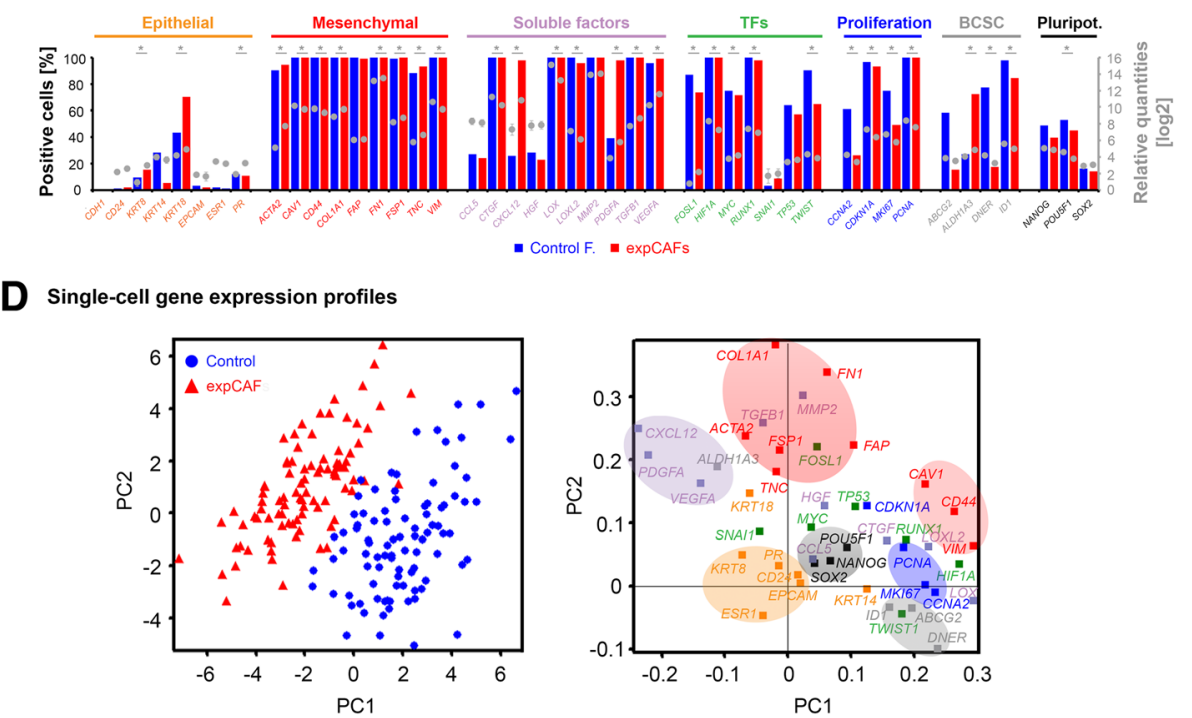

E ACTA2 distribution

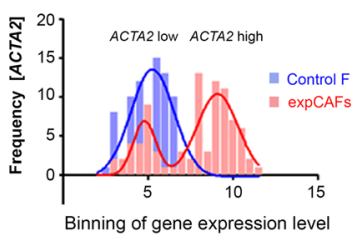

F ACTA2 stratification - gene correlations
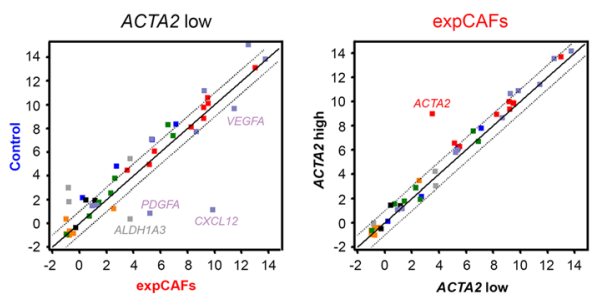

Fig. 1 Molecular markers distinguish normal fibroblasts from the tumour-activated counterparts. a Table of selected gene targets colour-coded according to their attributes (gene group). $\mathbf{b}$ Schematic representation of study design. $\mathbf{c}$ Basic statistics of single-cell gene expression profiles of experimental fibroblast model. Graphs represent frequency of selected gene targets in percentage as bars and average gene expression levels given as log2-transformed relative quantities depicted as dots. Error bars represent SEM ( ${ }^{*} p$-value < 0.05 Student $t$-test, Control F: control fibroblast, expCAF: experimentally-generated cancer-associated fibroblast). d (Left panel) Principal component analysis (PCA) of 183 individual Control fibroblasts ( $n=92$, blue dots) and expCAFs ( $n=91$, red triangles). In the principal component projections the position of a single cell (scores) is based on the expression of the analysed genes $(n=43)$, each dot represents a single cell. (Right panel) Plot represents the gene loadings for the PCA. Principal component projection of the genes illustrates the contribution of each gene to the scores of PC1 and PC2. Groups of genes are indicated as follows; orange: epithelial, red: fibroblast markers, purple: chemokines, green: transcription factors, blue: proliferation markers, grey: breast cancer-specific stem cell markers, black: pluripotency. e Histogram of distribution of ACTA2-specific gene expression with Gaussian regression analysis for control fibroblasts (blue line) and expCAFs (red line: sum of two Gaussians) demonstrating a bimodal gene expression pattern. f Plots depict correlations of gene expression between ACTA2 low expressing expCAFs and Control fibroblasts (left) and ACTA2 high expressing expCAFs (right). Genes outside the significance area (dotted black line) are denoted

Gene correlation analysis confirmed observed PCAprojected gene clusters and highlighted five main clusters (Fig. 2a). Gene cluster A displays a strong correlation of CAV1, CD44 and VIM with proliferation and BCSC markers. Strikingly, gene cluster B reveals correlation of all pluripotency markers with CCL5, which encodes for Rantes, a known mesenchymal stem cell effector [12] suggesting a distinct subpopulation with potential stem-like 


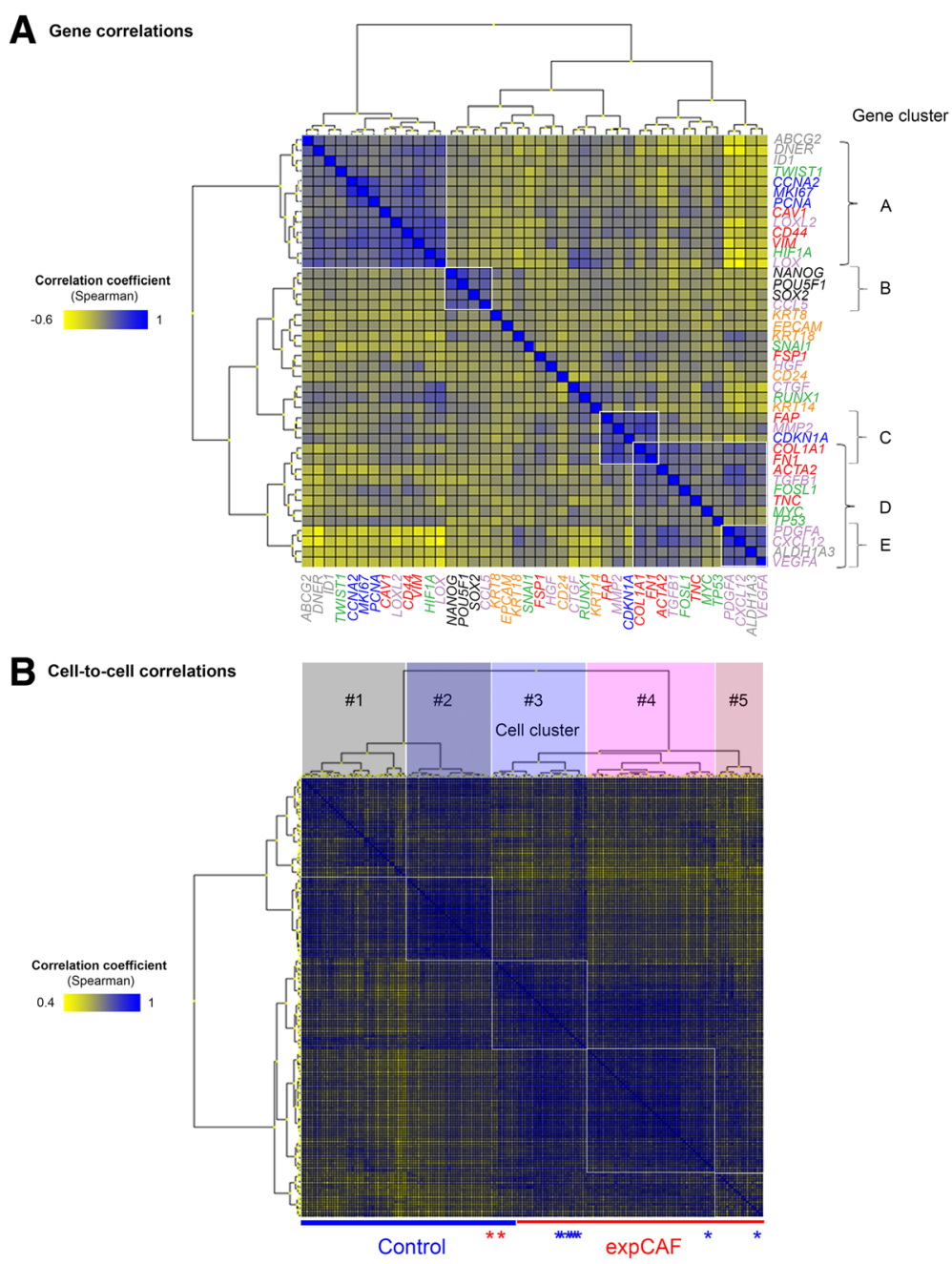

Fig. 2 Gene and cell-to-cell correlation analysis. a Heatmap (unsupervised clustering, Euclidian distance) demonstrating gene correlations coefficients (Spearman) based on gene expression of all cells. b Heatmap (unsupervised clustering, Euclidian distance) demonstrating cell-to-cell correlations coefficients (Spearman) based on individual gene expression profile of each cell. Manual grouping was performed according to hierarchical clustering to obtain five subgroups

properties. The remaining clusters display overlap of correlation, fibroblast markers FAP, COL1A1 and FN1 (Cluster C) correlate with ACTA2 and TNC (Cluster D) and to Cluster E including PDGFA, CXCL12, VEGFA and $A L D H 1 A 3$. The latter interestingly shows a strong inverse correlation with gene cluster A. Remarkably when analysing gene correlation separately, correlating gene clusters including pluripotency were largely maintained within normal fibroblasts but either overlapping or absent in CAF cells (Additional file 2A, middle and left panel). This data indicates the presence of a stem-like subtype within normal fibroblasts and that CAFs may be more streamlined or in fact displaying transitional phenotypes rather than true (distinct) subtypes.

Collectively, our study confirms a distinct regulation of fibroblast-specific markers, including several indicators for aggressiveness, making them suitable markers for monitoring fibroblast activation. Whether downregulated expression of mesenchymal markers is a side-effect or a fundamental step of fibroblast activation needs to be further tested.

\section{Identification and modelling of subpopulations defining fibroblast differentiation states}

To examine the existence of subpopulations at the single-cell level we performed cell-to-cell correlation analysis based on all analysed genes. The correlation coefficient (Spearman rho) which indicates how one cell relates to another is depicted in a heatmap, clustering cells by similarity (Fig. 2b). Cells were grouped according to hierarchical clustering of their correlation and consequently divided into five subgroups (Cell cluster \#1-5) including two groups per cell type and one mixed subgroup (Cell cluster \#3). Cell identification for clusters 
were noted and used for manual subgrouping of cells. In order to avoid skewing data by global RNA expression level, samples were normalized by autoscaling data per cell. As a result PCA analysis depicts clustering of identified cellular subgroups and also distinction of CAFs from normal counterparts (Fig. 3a). Strikingly, within cell cluster \#3 normal fibroblasts show gradual conversion to more CAF-like phenotype. Similar cell clusters were observed when subgrouping samples applying selforganizing map (SOM) analysis. Projection of gene loadings for normalized cells was principally unaffected, displaying similar gene groupings (Fig. 3a).

For further analysis of frequency and average expression levels of gene targets per cell group, we divided mixed cell cluster \#3 into control fibroblasts and expCAFs to assess potential differences in physiological fibroblast differentiation status and tumour-mediated fibroblast activation (Fig. 3b). We noticed that gene expression was mostly regulated by in- or decreased transcript level except for HGF, CCL5, pluripotency and BCSC-like target genes. Changes in number of positive cells may be indicative of a more switch-like gene regulatory mechanism of putative stem cells. To account for switch-like and transitional gene regulation equally, we generated pseudo-temporal gene expression profiles combining target-specific frequencies and relative expression levels for each cell cluster. Gene groups or similar gene expression profiles within the fibroblast and soluble factors gene transcripts are plotted together (Fig. 3c). According to their most prominent gene transcription we denoted normal fibroblast cell clusters as following; \#1 as 'stem-like' (defined by high pluripotency expression), \#2 'naive' (lowest activation marker expression) and \#3 as 'primed' (characterized with increased MMP2, FSP1, TNC, COL1A1 and FN1 expression) whereas expCAFs were denoted \#4 as 'protomyofibroblasts' (ACTA2 expression is higher compared to control fibroblasts, but lowest amongst CAFs), \#5 as 'ECM-regulating myofibroblasts' (highest COL1A1 and FN1 expression) and \#6 as 'secretory myofibroblasts' (highest CXCL12 and PDGFA expression). Based on selected markers unsupervised hierarchical clustering confirms much closer resemblance between 'stem-like' and 'naive' fibroblasts whereas 'primed' normal fibroblast were somewhat closer related to expCAFs, although still low in soluble factor secretion. According heatmap further illustrates proposed stepwise regulation of gene expression during fibroblast activation (Fig. 3d).

Overall, identifying distinct but overlapping gene expression patterns in fibroblast subpopulations allowed modelling of a transitional, progressive differentiation process and the regulation of pluripotency markers indicates an underlying hierarchical program.

\section{Molecular classification of patient-derived fibroblasts}

With regard to the CAF model, patient-derived normal and according cancer-associated fibroblasts revealed a conserved gene expression, defined by increased transcript levels of ACTA2, COL1A1, TNC, VEGFA and $A L D H 1 A 3$, with concomitant decreased expression of CAV1, CD44 and VIM in CAFs (Fig. 4a).

Likewise, PCA of primary normal fibroblasts and CAFs presents them as distinct molecular cell types (Fig. 4b). The according gene expression pattern was somewhat more convoluted, yet proliferation and pluripotency gene clusters remained. Identified gene clusters of cell line model are highlighted. We observed gene cluster A to be divided into two (A1, A2) marking normal fibroblasts and overlapping gene clusters C-D characterizing fibroblast activation and CAF phenotypes whereas gene cluster $\mathrm{E}$ is absent. In order to identify whether and which fibroblast markers contribute the most to a generic CAF phenotype, we combined both data sets, CAF model and primary fibroblasts, by mean-centering data. We found ACTA2, TNC and ALDH1A3 to be the best common CAF markers, however not sufficient for a clear distinction from normal fibroblasts (Fig. 4c). SMA $\alpha$ and Tenascin- $\mathrm{C}$ are known fibroblast activation and CAF markers [4], but to the best of our knowledge this is the first report linking an aldehyde dehydrogenase family member to CAF activation.

No clear distinct gene correlations defining normal and activated fibroblasts could be identified for primary cells (Additional file 2B, left panel). Gene cluster A and a minimized gene cluster $\mathrm{C} / \mathrm{D}$, attributed to 'naive' and 'proto-myofibroblastic' states respectively, were identified (Additional file 2B, middle and right panel). Individual gene correlations only hint at a stem cluster exclusive for the normal fibroblasts and a tighter gene co-regulation for CAFs. In line with PCA analysis no gene cluster $\mathrm{E}$ was observed, however we further observed an additional correlation cluster including TNC and VEGFA which was absent for normal fibroblasts (Additional file 2B). It has been suggested that FSP1positive CAFs expressing Tenascin $C$ and VEGFA represent metastasis-associated fibroblasts [13]. However analysed patient-derived CAFs revealed a pronounced decrease in FSP1 transcript levels.

Utilizing defined molecular fibroblast differentiation states we applied data of primary cells as test set within a PCA to determine their activation status. We found normal fibroblast to be closest resembling the "naive" phenotype, if somewhat more diverse, ranging from 'stem-like' to 'primed' than the CAFs which clustered more tightly mainly between the 'primed' and 'protomyofibroblastic' phenotypes. Thus, generation of a molecular differentiation model allows classification of patient-derived breast cancer-associated fibroblasts as 
A Cellular subpopulations
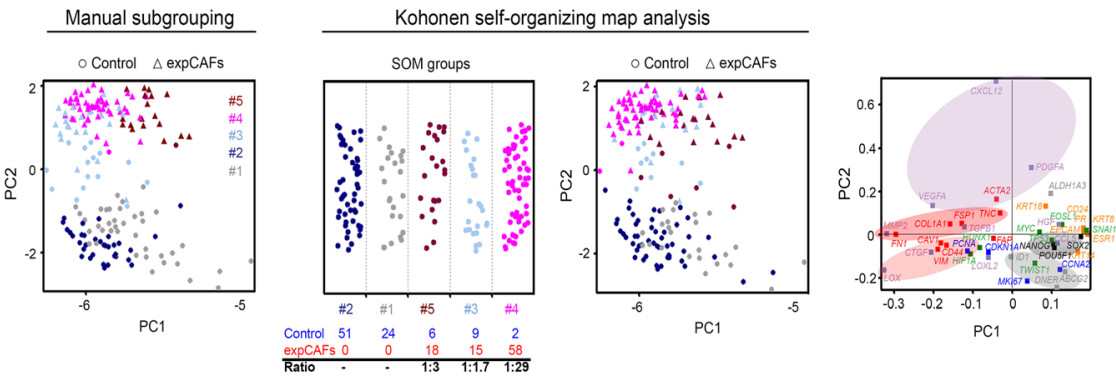

B Frequency and expression level
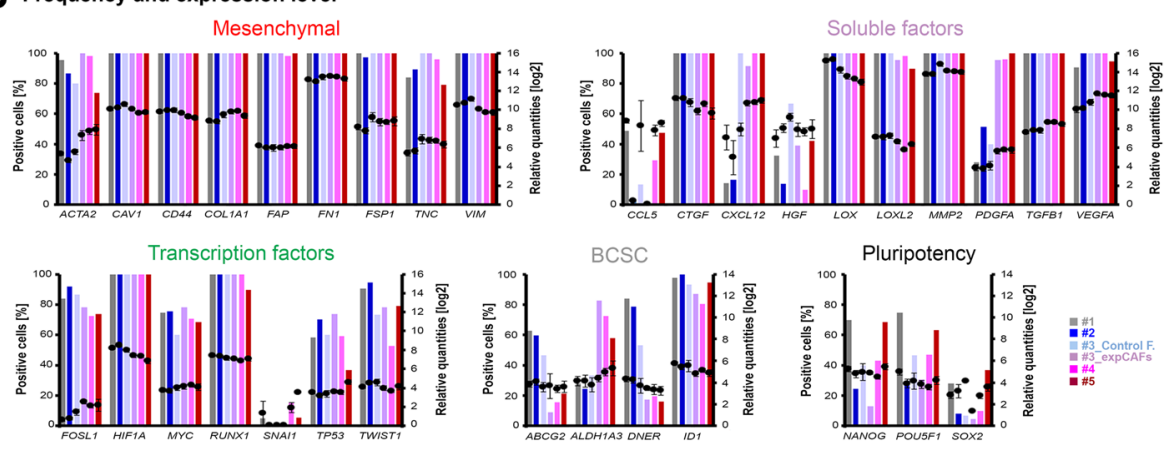

\section{Pseudo-temporal gene profiles (frequency and expression)}

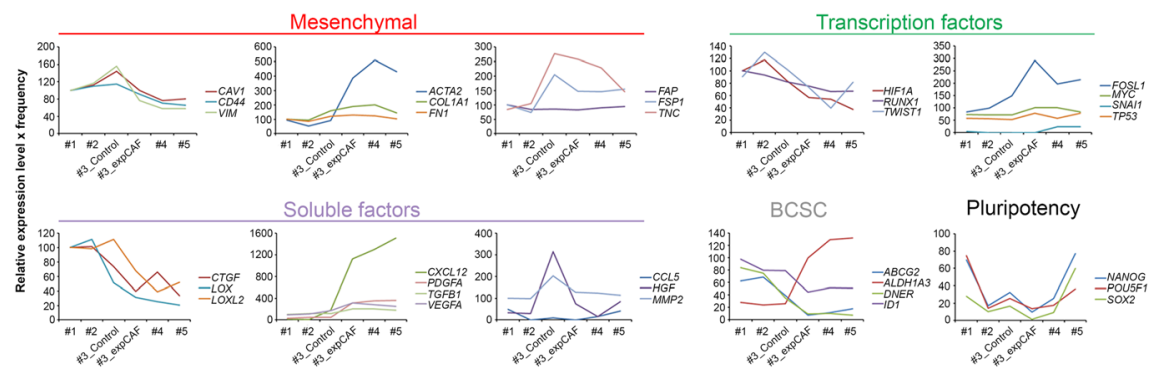

D Pseudo-temporal molecular fibroblast differentiation model

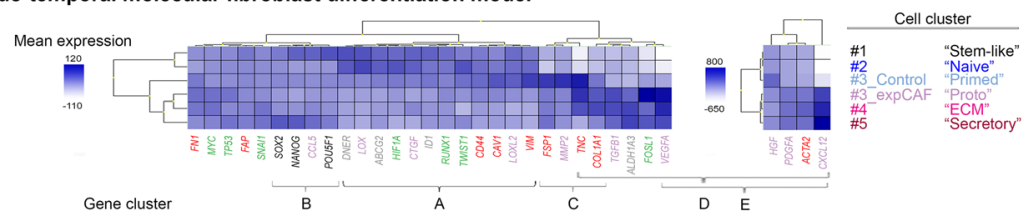

Fig. 3 Identification of fibroblast subpopulations and generation of a molecular differentiation model. a Principal component analysis (PCA) of 183 individual normal ( $n=92$, dots) and experimentally generated cancer-associated fibroblasts (expCAFs, $n=91$, triangles) colour-coded according to their subgroups. Data was normalized by autoscaling data per cell to eliminate differences due to global expression level (left). Kohonen self-organizing map (SOM) analysis confirms subgroups based on cell-to-cell correlation (middle). According gene loadings for PCA. Gene clusters are highlighted (right). $\mathbf{b}$ Basic statistics of single-cell gene expression profiles with regard to each subgroup. Bar graphs represent frequency of selected gene targets in percentage and average gene expression levels given as log2-transformed relative quantities are depicted as dots. Error bars represent SEM. c Graphs depict pseudo-temporal gene expression profile representing the product of relative expression level and frequency across subgroups. $\mathbf{d}$ Heatmap (unsupervised clustering) of pseudo-temporal gene profiles (combined frequency and relative average expression) per subgroup which were assigned a specific cellular state according to their most prominent gene transcripts thereby creating a pseudo-timeline of molecular differentiation states. Genes with extremely pronounced differences between subgroups were plotted separately for better visualization

early onset CAFs defined by relatively low ACTA2 positivity and moderate secretory profile and mostly exhibiting ECM remodelling qualities with high COL1A, TNC, MMP2, LOX and LOXL2 levels with concomitant decrease in $C A V 1$ and $C D 44$.

\section{Functional analysis of stem potential}

Stem cells are unspecialised cells at the apex of a hierarchical cellular organization with the ability to selfrenew and give rise to more specialised/ differentiated progeny. The discovery of specific markers and 
A Patient-derived fibroblasts: Frequency and expression level

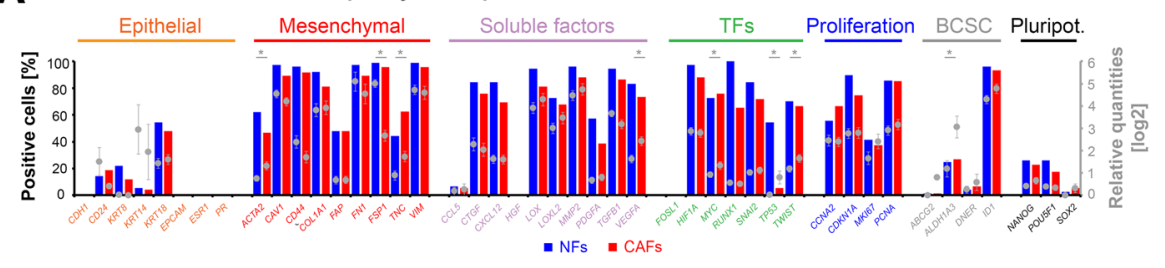

B Single-cell gene expression profile

C Combined data sets
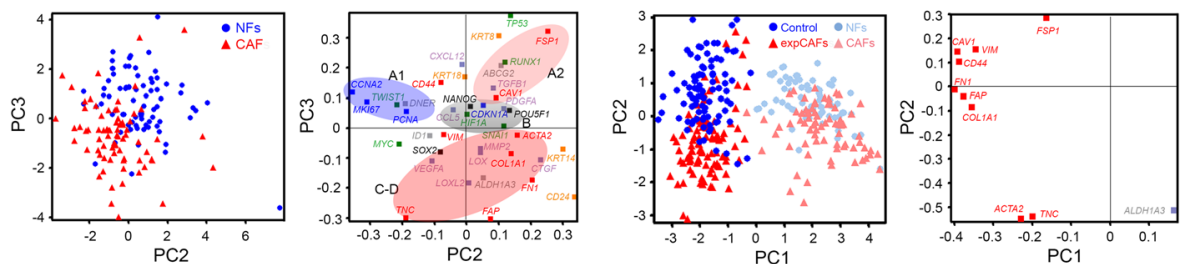

D Proof-of-principle: Fibroblast classification
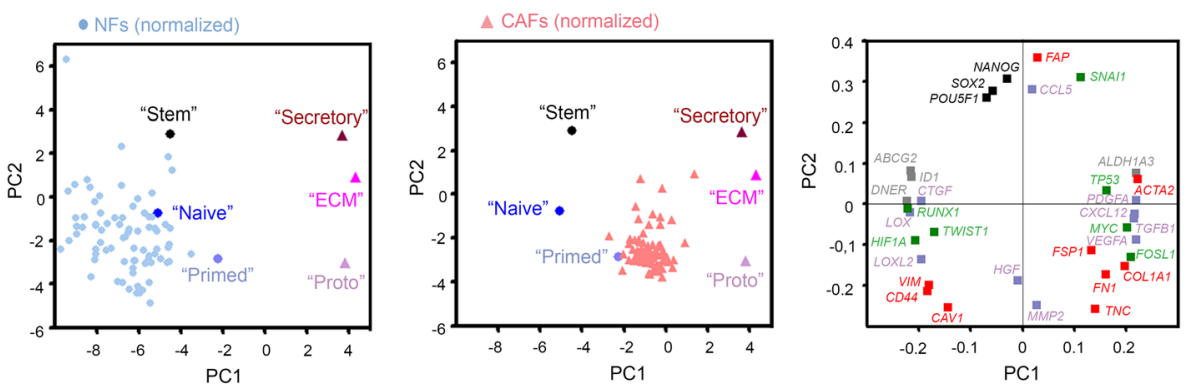

Fig. 4 Analysis of patient-derived fibroblasts. a Basic statistics of single-cell gene expression profiles of patient-derived fibroblasts isolated from invasive, ERa-positive breast cancer and according normal tissue. Graphs represent frequency of selected gene targets in percentage as bars and average gene expression levels given as log2-transformed relative quantities depicted as dots. Error bars represent SEM $\left({ }^{*} p\right.$-value $<0.05$ ANOVA, NF: normal fibroblast, CAF: cancer-associated fibroblast). b (Left pane) Principal component analysis (PCA) of 152 individual normal ( $n=77$, blue dots) and cancer-associated fibroblasts ( $n=75$, red triangles). (Right panel) Plot represents the gene loadings for the PCA. Principal component projection of the genes illustrates the contribution of each gene to the scores of PC2 and PC3. Groups of genes are indicated as follows; orange: epithelial, red: fibroblast markers, purple: chemokines, green: transcription factors, blue: proliferation markers, grey: breast cancer-specific stem cell markers, black: pluripotency. Identified gene clusters of cell line model are highlighted with gene cluster A divided into two (A1, A2) whereas gene cluster E is absent. c PCA depicting combined mean-centered datasets of single-cell gene expression of CAF model and patient-derived fibroblasts based on ten genes with potential fibroblast activation-predictive qualities. $\mathbf{d}$ PCAs of subgroups/differentiation states and applying patient-derived normal (left panel) and cancer-associated fibroblast (middle panel) single-cell data as test set. Patient data was normalized due to variable global expression levels by autoscaling data per cell. (Right panel) According gene loadings for PCAs

common molecular process underlying the core stem cell properties, also referred to as stemness, has been object of intense study. Stemness potential is typically characterized by high expression of pluripotency genes, tissue-specific stem cell markers and low proliferation rate/quiescence [14].

Cellular organization of fibroblasts is still obscure owing to diverse cells of origin and modes of activation. In order to validate the existence of a potential hierarchical framework of fibroblast we assessed anoikis resistance/anchorage independence and subsequent sphere formation capacity as functional readout for stem-like characteristics. Label-retention analysis was employed as proof-of-principle confirming the ability of a single cell to give rise to spheroid growth upon survival under non-adherent conditions (Fig. 5a). Furthermore, when we compared RNA expression of conventionally cultured fibroblasts with $24 \mathrm{~h}$ anoikis-resistant cells, we noted increased expression of all pluripotency genes and CCL5 while proliferation genes (CCNA2, MKI67) were downregulated (Fig. 5b). In line, CDKN1A, encoding p21 which regulates cell cycle arrest/ quiescence was upregulated. Remarkably, fibroblast activation markers ACTA2, $T N C$ and $A L D H 1 A 3$ showed lower expression whereas ECM markers COL1A1 and FN1 and several soluble factors (most prominently HGF, MMP2) exhibited higher transcript levels. Taken together, single-cell derived sphere formation and anoikis resistance-mediated increase in pluripotency support validity of our approach to monitor stemness.

Quantifying stem cell-like potential in the fibroblast cell lines we observed significant lower sphere number 
A Label-retention: Single-cell derived sphere
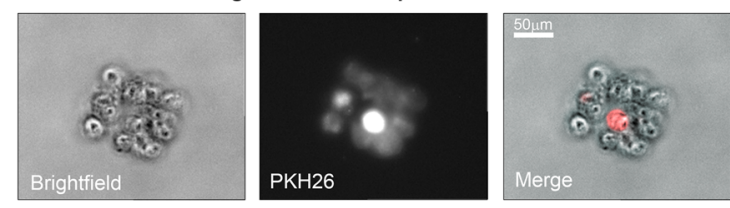

C Sphere formation

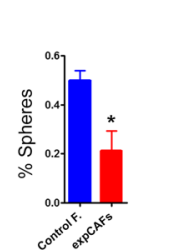

Fibroblast activation
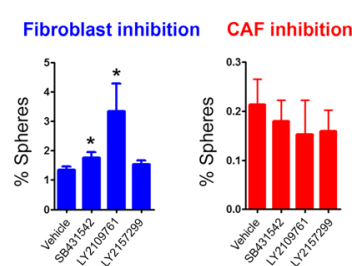

D Patient-derived fibroblasts: Sphere formation
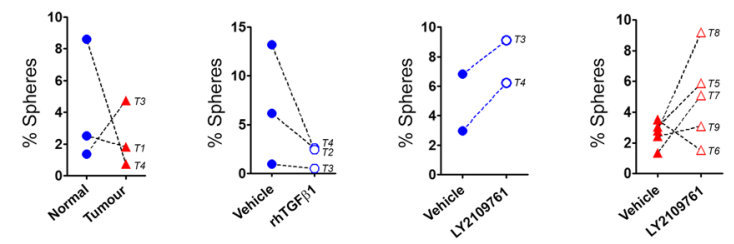

\section{B}

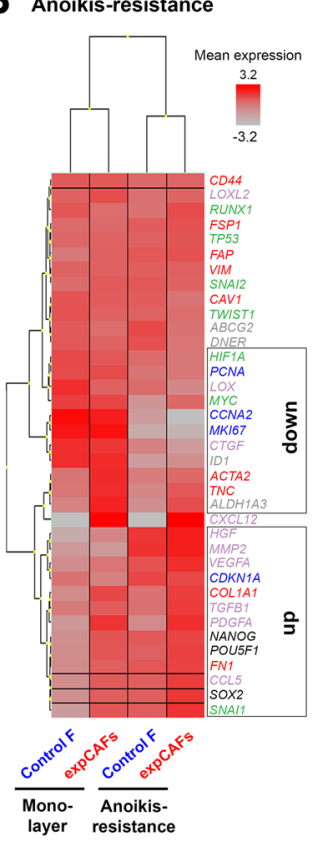

E Model: Fibroblast differentiation

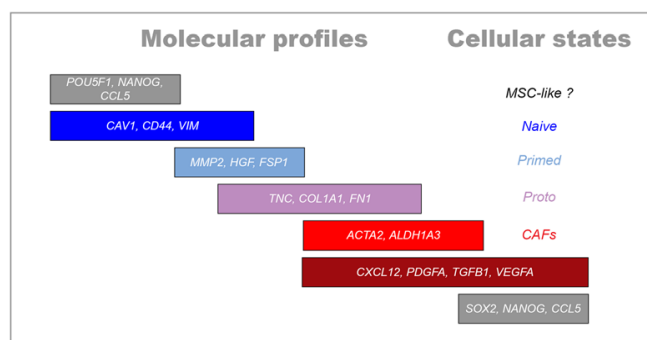

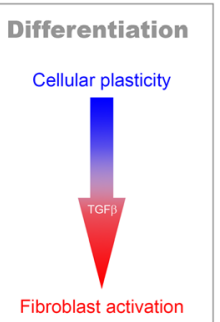

Fig. 5 Anoikis-resistance and sphere formation of fibroblast cells. a Microscopic picture depicting a sphere derived from a PKH-labelled single cell after 5 days in non-adherent culture conditions. b Heatmap (unsupervised clustering) of bulk RNA expression levels before and after $24 \mathrm{~h}$ of anoikis resistance. $\mathbf{c}$ Graphs represent sphere formation capacity as percentage of control (blue) and expCAFs (red) without and upon treatment with $10 \mathrm{ng} / \mathrm{ml}$ of recombinant human TGF $\beta 1$ (rhTGF $\beta 1$ ), $72 \mathrm{~h}$ cell-conditioned media of breast cancer cell lines (MCF7, MDA-MB-231) or 10uM of TGF $\beta$ signalling-targeted pharmacological inhibitors (TGFBR1 inhibitors: SB431542, LY2157299; dual TGFBR1/TGFBR2 inhibitor: LY2109761). Three independent experiments with triplicates were performed. $\mathbf{d}$ Graphs representing sphere formation as percentage of individual patient-derived fibroblasts (triplicates) depicted as normal-matched (blue) and cancer-associated fibroblast (red) and paired treatment response. Each sample is marked with corresponding tumour number (T1-T9). Clinical information for each tumour can be found in Additional file 1. e Working model of hierarchical fibroblast differentiation comprising diverse activation states with overlapping molecular profiles

for expCAFs (Fig. 5c). To test whether paracrine activation of fibroblasts accounts for loss of sphere-forming capacity we transdifferentiated control fibroblast through cultivation with recombinant human TGF $\beta 1$ ligand or cancer cell-conditioned media. TGF $\beta 1$ and cancersecreted factors significantly reduced sphere formation whereas the use of pharmacological TGF $\beta$ inhibitors increased sphere formation. This potentially highlights a key role of the TGF $\beta$ pathway in stem cell maintenance. In contrast inhibiting TGF $\beta$ pathway in expCAFs did not affect sphere numbers suggesting an irreversible differentiation state and diminished cellular plasticity of highly activated fibroblasts.
Two out of three patient-derived fibroblasts confirmed reduced sphere-forming capacity in CAFs (Fig. 5d). It is noteworthy that sphere number was considerably higher in primary cells compared to CAF model cell lines. Modulation of TGF $\beta$ signalling demonstrated reduced sphere formation in normal fibroblasts using TGF $\beta$ ligand and increased sphere number in both, normal fibroblasts and CAFs, upon TGF $\beta$ inhibition. Latter observation may be a result of either a retained cellular plasticity of ex vivo fibroblasts, distinct tumour-stromal interaction with the associated tumour or a mixed population of CAFs with discrete anatomic origin. It has been demonstrated that fibroblast isolated from distinct 
tumour zones exhibit different biomodulatory properties [15]. The recruitment process and functional role of MSCs to the tumour site is still matter of debate [16], however it was shown that about one fifth of CAFs may originate from bone-marrow derived MSCs [17]. Multipotency may thus be distinct from CAFs of other origin. Analysis of more patient samples is required to address this. Overall our data highlight the existence of a subset of cells with stem-like characteristics and suggest a hierarchical cellular organization of fibroblasts.

\section{Discussion}

CAFs are critical components of the tumour stroma and thus present a promising novel treatment option. However, the multifunctional role of CAFs and their fundamental heterogenic nature makes targeting the tumour stroma clinically challenging. Therefore it is essential to characterize underlying molecular processes of tumourassociated fibroblast activation in order to identify relevant markers enabling monitoring and appropriate targeting of potential functionally distinct CAF subtypes.

Within this comprehensive program we demonstrate that normal fibroblasts and their tumour-activated counterparts are molecularly distinct cell types. Beside loss of Caveolin-1 and increased SMA $\alpha$ and Tenascin-C, we report a member of the ALDH family as a novel fibroblast activation marker which may be linked to pro-tumourigenic features such as secretion of angiogenic and chemotactic growth factors (ie. VEGFA, PDGFA, SDF1). We suggest a combination of these markers to determine stromal activation status as no single marker accounts for all CAFs.

We further reveal that CAFs differentiate with simultaneous increase of numerous characteristic fibroblast activation target genes and paracrine factors with a dominating group of genes regulating a specific tumour microenvironmental aspect such as ECM remodelling ('Type I') or growth factor/ cytokine secretion ('Type II'). Based on cell-to-cell similarity and the overlapping gene expression profiles indicating transitional states, we created subgroups in a sequential manner. Generating a pseudo-timeline recapitulating theoretical differentiation stages allowed ultimately for classification of patientderived fibroblasts to be predominantly of an early-stage phenotype characterized with minimal ACTA2 induction but ECM-remodelling qualities ('proto-myofibroblast') (Fig. 4d).

Notably, an earlier study suggested two distinct CAF subtypes based on either SMA $\alpha$ or FSP1 expression [18]. Both markers have been shown to be protumourigenic. However FSP1 was found to be expressed on resting as well as activated fibroblasts. With regard to our data highlighting FSP1 as a marker for a 'primed' cellular state found in normal fibroblasts, we suggest FSP1 as an early marker in fibroblast activation that may be reverted upon SMA $\alpha$ induction. In accordance, we observed FSP1 profoundly reduced in the patient-derived CAFs compared to the normal counterparts whereas SMA $\alpha$ encoding ACTA2 gene expression level increased together with TNC and ALDH1A3. Loss of FSP1 in mice was shown to impair fibroblast motility and reduced metastasis. Strikingly, in line with this, evaluation of clinical aspects of analysed tumour revealed the absence of lymph node metastasis despite the high risk of lymph node involvement for the ER $\alpha+/ \mathrm{HER} 2+/ \mathrm{Ki} 67^{\text {high }}$ tumour subtype [19].

In more detail, analysed fibroblasts are derived from a patient with invasive ductal carcinoma molecularly characterized with high Ki67 index, ER $\alpha$ - and PR-positivity and harbouring HER2 amplification (Additional file 1). This expression profile classifies the tumour to be of the luminal B subtype with worse prognosis compared to the ER $\alpha$-positive luminal A subtype [20]. Further, the histopathologically observed vascular invasion is associated with unfavourable outcome [21]. However, the tumour presented with residual DCIS which was shown to be associated with favourable outcome [22] and most importantly the tumour displayed no lymph node involvement which is most significant prognostic indicator for patients with early-stage breast cancer [21].

Taking together the tumours clinico-pathological parameters and CAFs' gene expression profiling it is tempting to assume that despite aggressive features such as big tumour size, high Ki67 status and vascular invasion, that the presence of DCIS, absence of lymph node metastasis and lack of chemotactic "secretory type II CAFs" marks the onset of a spreading tumour which has not yet manifested. With this in mind, analysing CAF-specific markers could be of great prognostic and/ or predictive value aiding patient selection and thus advancing personalised medicine.

Our study further highlights the existence of stem-like cells as a physiological feature of tissue-resident fibroblasts. Putative stem-like cells were defined as expressors of pluripotency genes and surviving non-adherent conditions which induces or enriches for pluripotency gene expression, and give rise to single-cell derived spheres. Strikingly, pluripotency correlated with CCL5 expression, which is a prominent factor in stromal gene expression signatures and linked to worse prognosis in breast cancer $[12,23]$. It has previously been demonstrated that fibroblastic cells within the tumour stroma possess mesenchymal stem cell (MSC) qualities and that breast cancer cells stimulate secretion of CCL5, which in turn facilitates its metastatic capacity [12]. Fibroblasts are likely to be more restricted in their differentiation potential than multipotent MSCs, but they may retain some cellular plasticity which appears to be regulated by 
TGF $\beta$. Very few studies have reported an anoikisresistant phenotype of fibroblasts, however with a protective role of TGF $\beta$ on survival of differentiated fibroblasts $[24,25]$ which may be a distinct functional feature compared to assessing single-cell survival with subsequent sphere formation. Notably, CAFs appear to be associated with a loss in sphere-forming capacity potentially representing a more differentiated cell type with diminished regenerative ability or cellular plasticity. However it remains to be determined how stem-like CAFs differ functionally from normal stem-like fibroblasts and whether their regulation is dependent on the associated tumour type or other microenvironmental factors.

Of note, a recent review by Kalluri suggests that resting or quiescent fibroblasts may be considered adult tissueresident mesenchymal stem cell [26]. Even further our data presented here is in line with the hypothesized three main fibroblast phenotypes: (1) resting or quiescent fibroblasts with MSC capacity, (2) normal or wound-healingassociated activated fibroblasts (NAFs) with increased ECM synthesis, secretion and motility thus representative of "primed" fibroblasts and (3) CAFs which exert an enhanced secretory profile while concomitantly associated with loss of contractility (SMA $\alpha$ ) and decreased synthetic activity (collagen, fibronectin) compared to NAFs. However transitional stages or cellular states need to be further defined.

\section{Conclusion}

Collectively our data indicate that underlying the cellular heterogeneity, fibroblasts may be hierarchically organized (see model Fig. 5e). The notion of cancer as "a wound that never heals" implies parallels of the transcriptional program between fibroblasts in the tumour stroma and during wound response. We propose that tumour-secreted factors such as TGF $\beta$ recruit and activate resident 'naive' fibroblasts. A subsequent increase in ECM deposition and ECM-remodelling enzymes is 'priming' fibroblasts to a more differentiated state whereas loss of particular mesenchymal markers such as Caveolin-1 may indicate a less reversible commitment of fibroblast activation. This ultimately results in archetypical CAFs with excessive matrix production ('Type I') and secretion of angiogenic and metastatic growth factors and immunomodulatory cytokines ('Type II'). During the fibroblast differentiation, physiological stem or regenerative potential may be lost, mediated by tumour-secreted factors such as TGF $\beta$ which in turn effectively induces fibroblast activation.

Distinct fibroblast specialization may depend on cell of origin [27] and it has been noted that heterogeneity and interactions of different types of CAFs potentiates tumour-promoting qualities [28]. Findings reported herein relate to the ER $\alpha$-positive breast cancer subtype and tissue-resident derived fibroblasts. It has been noted that the majority of CAFs are derived from local stem or progenitor cells whereas about a third may have different cellular origins [28]. We present evidence that tissuederived fibroblastic cells provide a local source for CAFs and contribute to the immunomodulatory function of the reactive tumour stroma. Overlapping marker expression pattern may indicate transitional cellular states rather than distinct cell types and predominant cellular states of CAFs may be inherent or affected by extrinsic factors such as genetic makeup of the tumour or tumour stage.

Further characterization of individual tumour stroma phenotypes will expand opportunities to discover novel clinical biomarkers and therapeutic targets. Categorizing patient groups with respect to CAFs in combination with current diagnostic tests will enable a more reliable selection of therapeutic strategies that block the tumour microenvironment.

\section{Additional files}

Additional file 1: Characterization of patient-derived fibroblasts and clinical information of used tumours. Fibroblast morphology and SMAa expression levels of isolated normal and cancer-associated fibroblasts. Clinical information of all analysed tumours. (PDF $1033 \mathrm{~kb}$ )

Additional file 2: Gene correlation analysis. Heatmaps depicting gene correlation analyses according to fibroblast subgroups (normal versus cancer-activated) for CAF cell line model and primary fibroblasts. (PDF 2716 kb)

\section{Abbreviations}

7AAD: 7-amino-actinomycin D; ABCG2: Adenosine triphosphate-binding cassette sub-family G member 2; ACTA2: Actin, alpha 2, smooth muscle, aorta; ALDH1A3: Aldehyde dehydrogenase 1 family member A3; ANOVA: Analysis of variance; BCSC: Breast cancer-specific stem cells; BSA: Bovine serum albumin; CAFs: Cancer-associated fibroblasts; CAV1: Caveolin 1; CCL5: C-C motif chemokine ligand 5; CCNA2: Cyclin A2; CD24: Cluster of differentiation 24; CD44: Cluster of differentiation 44; $\mathrm{CDH1}$ : Cadherin 1; CDKN1A: Cyclin dependent kinase inhibitor 1A; CDNA: Complementary deoxyribonucleic acid; $\mathrm{CO}_{2}$ : Carbon dioxide; COL1A1: Collagen, type I, alpha 1; CTGF: Connective tissue growth factor; CXCL12: C-X-C motif chemokine ligand 12; DNA: Deoxyribonucleic acid; DNase: Deoxyribonuclease; DNER: Delta/notch like epidermal growth factor repeat containing; ECM: Extracellular matrix; EPCAM: Epithelial cell adhesion molecule; ERa: Oestrogen receptor alpha; ESR1: Oestrogen receptor 1; expCAFs: Experimentally generated cancer-associated fibroblasts; FACS: Fluorescence-activated cell sorter; FAP1: Fas-associated phosphatase 1; FBS: Foetal bovine serum; FN1: Fibronectin 1; FOSL1: Fos-like antigen 1; FSP1: Fibroblast-specific protein 1; GAPDH: Glyceraldehyde-3-phosphate dehydrogenase; HGF: Hepatocyte growth factor; HIF1A: Hypoxia-induced factor 1 alpha; ID1: Inhibitor of deoxyribonucleic acid binding 1; KRT14: Keratin 14; KRT18: Keratin 18; KRT4: Keratin 4; LOX: Lysyl oxidase; LOXL2: Lysyl oxidase like 2; MKI67: Marker of proliferation Ki-67; MMP2: Matrix metallopeptidase 2; MSC: Mesenchymal stem cell; NANOG: Nanog homeobox; PCA: Principle component analysis; PCNA: Proliferating cell nuclear antigen; PCR: Polymerase chain reaction; PDGFA: Platelet-derived growth factor; PGR: Progesterone receptor; poly-HEMA: Poly-2-hydroxyethyl methacrylate; POU5F1: POU class 5 homeobox 1; RNA: Ribonucleic acid; RNase: Ribonuclease; RT-PCR: Reverse transcriptase polymerase chain reaction; RUNX1: Runt-related transcription factor 1; SEM: Standard error of mean; SMAa: Smooth muscle actin alpha; SNAI1: Snail family transcriptional repressor 1; SNAI2: Snail family transcriptional repressor 2; SOM: Kohonen self-organizing maps; SOX2: Sex determining region Y-box 2; 
TGFB1: Transforming growth factor beta 1; TGF $\beta$ : Transforming growth factor beta; TNC: Tenascin C; TP53: Tumor protein p53; TWIST1: Twist family basic helix-loop-helix transcription factor 1; VEGFA: Vascular endothelial growth factor A; VIM: Vimentin

\section{Acknowledgement}

The authors would like to thank Dr Akira Orimo for kindly providing us with the experimental CAF model cell lines. The authors would also like to thank Ylva Magnusson and Pernilla Gregersson for collection and handling of tissue samples and patient data.

\section{Funding}

This study was supported by the Swedish Research Council and the Swedish Cancer Society.

\section{Availability of data and materials}

The datasets generated during and analysed during the current study are available from the corresponding author on reasonable request.

\section{Authors' contributions}

SB was involved preparation and data acquisition of single-cell qPCR and performed cell-based assays, analysed and interpreted data and drafted manuscript. DA performed primer design and validation and single-cell GPCR. $\mathrm{EB}$ and $\mathrm{CW}$ performed fluorescence-activated cell sorting. AS and GL were involved in experimental set up, data analysis, interpretation and drafting of manuscript. All authors read and approved the final manuscript.

\section{Competing interests}

A.S. declares stock ownership in TATAA Biocenter.

\section{Consent for publication}

Not applicable.

\section{Ethics approval and consent to participate}

Processing of patient material and data has been approved by the Regional Research Ethics Committee (REC) in Gothenburg (reference number: 515-12).

\section{Publisher's Note}

Springer Nature remains neutral with regard to jurisdictional claims in published maps and institutional affiliations.

\section{Author details}

'Department of Pathology, Sahlgrenska Cancer Center, Institute of Biomedicine, Sahlgrenska Academy, University of Gothenburg, Gothenburg, Sweden. ${ }^{2}$ Department of Surgery, Institute of Clinical Sciences, Transplantation and Regenerative Medicine, Sahlgrenska Academy, University of Gothenburg, Gothenburg, Sweden.

\section{Received: 16 November 2016 Accepted: 22 March 2017}

\section{Published online: 03 April 2017}

\section{References}

1. Ohlund D, Elyada E, Tuveson D. Fibroblast heterogeneity in the cancer wound. J Exp Med. 2014:211:1503-23.

2. Dvorak HF. Tumors: wounds that do not heal. Similarities between tumor stroma generation and wound healing. N Engl J Med. 1986:315:1650-9.

3. Karagiannis GS, Poutahidis T, Erdman SE, et al. Cancer-associated fibroblasts drive the progression of metastasis through both paracrine and mechanical pressure on cancer tissue. Mol Cancer Res. 2012;10:1403-18.

4. Augsten M. Cancer-associated fibroblasts as another polarized cell type of the tumor microenvironment. Front Oncol. 2014;4:62.

5. Tchou J, Kossenkov AV, Chang $L$, et al. Human breast cancer associated fibroblasts exhibit subtype specific gene expression profiles. BMC Med Genet. 2012:5:39.

6. Busch S, Landberg G. CAF-specific markers: role of the TGFbeta pathway. Oncoscience. 2015;2:835-6.

7. Kojima Y, Acar A, Eaton EN, et al. Autocrine TGF-beta and stromal cellderived factor-1 (SDF-1) signaling drives the evolution of tumor-promoting mammary stromal myofibroblasts. Proc Natl Acad Sci U S A. 2010;107: 20009-14.
8. Akrap N, Andersson D, Bom E, et al. Identification of Distinct Breast Cancer Stem Cell Populations Based on Single-Cell Analyses of Functionally Enriched Stem and Progenitor Pools. Stem Cell Reports. 2016;6:121-36.

9. Stahlberg A, Rusnakova V, Forootan A, et al. RT-qPCR work-flow for singlecell data analysis. Methods. 2013;59:80-8.

10. Martinez-Outschoorn UE, Sotgia F, Lisanti MP. Caveolae and signalling in cancer. Nat Rev Cancer. 2015;15:225-37.

11. Yamashita M, Ogawa T, Zhang X, et al. Role of stromal myofibroblasts in invasive breast cancer: stromal expression of alpha-smooth muscle actin correlates with worse clinical outcome. Breast Cancer. 2012;19:170-6.

12. Karnoub AE, Dash AB, Vo AP, et al. Mesenchymal stem cells within tumour stroma promote breast cancer metastasis. Nature. 2007;449:557-63.

13. O'Connell JT, Sugimoto H, Cooke VG, et al. VEGF-A and Tenascin-C produced by S100A4+ stromal cells are important for metastatic colonization. Proc Natl Acad Sci U S A. 2011;108:16002-7.

14. Melton DA, Cowen C. "Stemness": Definitions, Criteria, and Standards. Essentials of Stem Cell Biology. 2nd ed. 2009. p. Xxiii-Xxix.

15. Gao MQ, Kim BG, Kang S, et al. Stromal fibroblasts from the interface zone of human breast carcinomas induce an epithelial-mesenchymal transitionlike state in breast cancer cells in vitro. J Cell Sci. 2010;123:3507-14.

16. Yagi $H$, Kitagawa $Y$. The role of mesenchymal stem cells in cancer development. Front Genet. 2013:4:261.

17. Quante M, Tu SP, Tomita H, et al. Bone marrow-derived myofibroblasts contribute to the mesenchymal stem cell niche and promote tumor growth. Cancer Cell. 2011;19:257-72.

18. Sugimoto $\mathrm{H}$, Mundel TM, Kieran MW, et al. Identification of fibroblast heterogeneity in the tumor microenvironment. Cancer Biol Ther. 2006;5:1640-6.

19. Inic Z, Zegarac $M$, Inic $M$, et al. Difference between Luminal A and Luminal B Subtypes According to Ki-67, Tumor Size, and Progesterone Receptor Negativity Providing Prognostic Information. Clin Med Insights Oncol. 2014; 8:107-11.

20. Sorlie T, Perou CM, Tibshirani R, et al. Gene expression patterns of breast carcinomas distinguish tumor subclasses with clinical implications. Proc Natl Acad Sci U S A. 2001;98:10869-74.

21. Cianfrocca M, Goldstein LJ. Prognostic and predictive factors in early-stage breast cancer. Oncologist. 2004;9:606-16.

22. Chagpar AB, McMasters KM, Sahoo S, et al. Does ductal carcinoma in situ accompanying invasive carcinoma affect prognosis? Surgery. 2009;146:561-7. discussion 567-568.

23. West RB, Nuyten DS, Subramanian $S$, et al. Determination of stromal signatures in breast carcinoma. PLoS Biol. 2005;3:e187.

24. Horowitz JC, Rogers DS, Sharma V, et al. Combinatorial activation of FAK and AKT by transforming growth factor-beta1 confers an anoikis-resistant phenotype to myofibroblasts. Cell Signal. 2007:19:761-71.

25. Kim BG, Gao MQ, Choi YP, et al. Invasive breast cancer induces laminin-332 upregulation and integrin beta4 neoexpression in myofibroblasts to confer an anoikis-resistant phenotype during tissue remodeling. Breast Cancer Res. 2012:14:R88.

26. Kalluri R. The biology and function of fibroblasts in cancer. Nat Rev Cancer. 2016:16:582-98

27. Gascard P, Tlsty TD. Carcinoma-associated fibroblasts: orchestrating the composition of malignancy. Genes Dev. 2016;30:1002-19.

28. Barron DA, Rowley DR. The reactive stroma microenvironment and prostate cancer progression. Endocr Relat Cancer. 2012;19:R187-204.

\section{Submit your next manuscript to BioMed Central} and we will help you at every step:

- We accept pre-submission inquiries

- Our selector tool helps you to find the most relevant journal

- We provide round the clock customer support

- Convenient online submission

- Thorough peer review

- Inclusion in PubMed and all major indexing services

- Maximum visibility for your research

Submit your manuscript at www.biomedcentral.com/submit
BioMed Central 